\title{
Learning from the past: development of safe and effective COVID-19 vaccines
}

\author{
Shan Su, Lanying Du and Shibo Jiang (D)
}

Abstract | The rapid spread of severe acute respiratory syndrome coronavirus 2 (SARS-CoV-2) has elicited an equally rapid response aiming to develop a COVID-19 vaccine. These efforts are encouraging; however, comprehensive efficacy and safety evaluations are essential in the development of a vaccine, and we can learn from previous vaccine development campaigns. In this Perspective, we summarize examples of vaccine-associated disease enhancement in the history of developing vaccines against respiratory syncytial virus, dengue virus, SARS-CoV and Middle East respiratory syndrome coronavirus, which highlight the importance of a robust safety and efficacy profile, and present recommendations for preclinical and clinical evaluation of COVID-19 vaccine candidates as well as for vaccine design and optimization.

Since December 2019, severe acute respiratory syndrome coronavirus 2 (SARS-CoV-2) has rapidly spread around the globe. The intensity and rapidity of SARS-CoV-2 transmission have led to substantial morbidity and mortality and put considerable pressure on public health systems around the world and the global economy. Consequently, developing vaccines and therapeutics against COVID-19 is of highest priority and a very active field ${ }^{1}$. Vaccines can prevent disease in large populations at relatively low cost, thus being a powerful tool to mitigate the impacts of COVID-19.

On 16 March 2020, the mRNA COVID-19 vaccine (mRNA-1273) from Moderna and the non-replicating adenovirus type 5 (Ad5)-vectored COVID-19 vaccine (Ad5-nCoV) from CanSino entered phase I clinical trials ${ }^{2,3}$. In April 2020, inactivated COVID-19 vaccines manufactured by Sinovac (PiCoVacc), the Beijing Institute of Biological Products (BBIBP-CorV) and the Wuhan Institute of Biological Products (Sinopharm-Wuhan inactivated vaccine), as well as Inovio's DNA vaccine (INO-4800), entered phase I clinical trials ${ }^{4}$. One month later, five more candidates had also entered phase I clinical trials, and more than 100 COVID-19 vaccine candidates were in laboratory or preclinical studies ${ }^{5}$. The unprecedented speed in the development of COVID-19 vaccines is encouraging. However, we and others have raised concerns about the safety of some of the COVID-19 vaccine candidates ${ }^{6,7}$.

A high dose of the mRNA-1273 vaccine protects mice against infection by mouse-adapted SARS-CoV-2 challenge without enhanced immunopathology ${ }^{8}$. PiCoVacc ${ }^{9}$ and BBIBP-CorV ${ }^{10}$ elicited neutralizing antibodies (NAbs) in mice, rats and non-human primates, and nonhuman primates in the high-dose group were fully protected from infection by SARS-CoV-2 with no antibody-dependent enhancement (ADE). The chimpanzee advenovirus-vectored vaccine developed by the University of Oxford and AstraZeneca (ChAdOx1 nCoV-19) ${ }^{11}$ and a DNA vaccine ${ }^{12}$ produced by Harvard Medical School were also effective in reducing viral load in SARS-CoV-2-challenged non-human primates without enhanced immunopathology. So far, several COVID-19 vaccine phase I/II clinical trials have been completed, including trials of Ad5-nCoV ${ }^{3}$, mRNA-1273 (REF. ${ }^{2}$ ), ChAdOx1 nCoV-19 $\left(\right.$ REF $\left.^{13}\right)$ and an mRNA vaccine developed by Pfizer and BioNTech $(\mathrm{BNT} 162 \mathrm{~b} 1)^{14}$. According to the reported results, all of these vaccines induced antibodies against the spike protein (S protein) and the receptor-binding domain (RBD), including antibodies that neutralized pseudotyped and live SARS-CoV-2. Some reports have shown that NAb titres were strongly correlated with the concentration of RBD-binding $\operatorname{IgG}^{15,16}$. Very recently, AstraZeneca announced a pause in the phase III clinical trial of its ChAdOx1 nCoV-19 vaccine because of an unexpected adverse reaction, although the trial has resumed in the United Kingdom. Furthermore, Russia recently approved a recombinant Ad26 and recombinant Ad5 vector-based heterologous primeboost COVID-19 vaccine for use in tens of thousands of people after conducting non-randomized phase I/II studies ${ }^{17}$. Vaccine safety remains a key question in phase III clinical trials and in the future application of vaccines, in particular for vaccine-related immunopathologies occurring when vaccinated people are naturally infected, as described below.

In the 1960s, scientists found that antiviral antisera might result in an exceptional increase in viral infectivity of animal viruses ${ }^{18}$. This phenomenon that viral infection can be enhanced by internalization associated with antibody Fc receptors (FcRs), denoted as 'antibody-dependent enhancement' (ADE; BOX 1), was then widely reported in infections with flaviviruses ${ }^{19,20}$ and other viruses ${ }^{21,22}$. Later, more antibody FcR-mediated effects, such as complement activation and release of inflammatory cytokines, were reported to be involved in severer disease ${ }^{23}$. ADE has also been observed in vaccinated animals after viral challenge with the corresponding virus $^{24}$. For example, cats immunized with a vaccine expressing the feline infectious peritonitis virus (FIPV) S protein on a recombinant pox virus vector died earlier than control animals when challenged with FIPV $^{25}$. Given that passive immunization with feline serum containing high-titre antibodies reactive with feline FIPV also resulted in a more rapid disease after FIPV challenge $^{26}$, the vaccine-induced disease exacerbation may be attributed to ADE. Apart from ADE, type $2 \mathrm{~T}$ helper cell $\left(\mathrm{T}_{\mathrm{H}} 2\right.$ cell)-based immunopathologic responses induced by homologous viral 


\section{Box $1 \mid$ Key terms in disease enhancement}

\section{ADE}

Antibody-dependent enhancement (ADE) can be mediated by antibody Fc receptor-associated internalization of a virus, thus resulting in more extensive viral replication and cytokine release in the presence of virus-specific antibodies. ADE was widely reported in flavivirus and other viral infections, such as HIV and influenza virus infections.

\section{ERD}

Enhanced respiratory disease (ERD) describes severer clinical symptoms after respiratory virus infection, such as with respiratory syncytial virus and influenza virus, due to previous immune responses. ERD usually manifests itself as peribronchiolar monocytic infiltration with an excess of eosinophils. ERD can happen during homotypic or heterotypic serotype virus infection after vaccination, natural infection or transfer of maternal passive immunity.

\section{VADE}

Vaccine-associated disease enhancement (VADE) partially overlaps with ADE and ERD. In contrast to ERD, VADE involves only the vaccine-associated situation, and, more importantly, it is not limited to respiratory disease. For example, heterotypic-serotype dengue virus infection may cause severer dengue haemorrhagic fever in vaccinated individuals. This phenomenon is related to VADE, but does not include ERD. VADE can be attributed to antibody-dependent and type 2 Thelper cell-dependent mechanisms.

challenge after vaccination could also result in disease exacerbation ${ }^{27}$.

In this Perspective, we use the term 'vaccine-associated disease enhancement' (VADE; BOX 1) to describe both antibodydependent and $\mathrm{T}_{\mathrm{H}} 2$ cell-dependent disease exacerbation (FIG. 1). We summarize examples of VADE in the history of the development of vaccines against respiratory syncytial virus (RSV), dengue virus (DENV), SARS-CoV and Middle East respiratory syndrome coronavirus (MERS-CoV), each of which provides clues for safe COVID-19 vaccine development and highlights the need for rigorous preclinical and clinical safety testing.

\section{Lessons from RSV vaccines}

There have been warnings that ADE should be fully evaluated for coronavirus vaccines to avoid repeating the tragic failure of the RSV vaccine ${ }^{28}$. The first RSV vaccine, based on formalin-inactivated RSV (FI-RSV), entered a clinical trial in 1965 , a time when several other inactivated or attenuated virus-based vaccines had already been successfully developed, such as vaccines against smallpox ${ }^{29}$ and polio ${ }^{30}$. The FI-RSV vaccine was well tolerated and appeared to be moderately immunogenic at first. However, instead of protecting study participants, the FI-RSV vaccine exhibited a paradoxical disease-strengthening effect (enhanced respiratory disease (ERD); BOX 1) during subsequent natural RSV infection. Among the 20 infants who received the FI-RSV vaccine, 16 required hospitalization, including two who subsequently died, whereas only one of the 21 participants in the control group was hospitalized $^{31}$. The FDA then urgently suspended all clinical studies of RSV vaccines.

To elucidate the mechanism of ERD in this RSV vaccine trial, the humoral and cellular immune responses after FI-RSV inoculation were analysed. FI-RSV induced RSV glycoprotein binding, but not NAbs, eosinophilia and an exaggerated $\mathrm{CD} 4^{+} \mathrm{T}$ cell response $^{32,33}$. It was not until the 1990s, three decades after the first FI-RSV trial, that an enhanced inflammatory response to the vaccine was identified, consisting of a $\mathrm{T}_{\mathrm{H}} 2$ cell-skewed $\mathrm{T}$ cell response, which contributed to the exaggerated proliferation of $\mathrm{CD}^{+} \mathrm{T}$ cells and eosinophils ${ }^{27,34,35}$. This $\mathrm{T}_{\mathrm{H}} 2$ cell-skewed pattern led to poor stimulation of natural killer cells and $\mathrm{CD}^{+}$cytotoxic $\mathrm{T}$ lymphocytes, which otherwise are able to prevent $\mathrm{T}_{\mathrm{H}} 2$ cell and inflammatory responses to RSV antigens ${ }^{36,37}$. Recent work suggested that the carbonyl groups caused by formalin fixation created the enhanced $\mathrm{T}_{\mathrm{H}} 2$ cell response ${ }^{38}$. However, ERD was also observed in experimental animals immunized with purified RSV $F$ and $G$ glycoproteins that were not fixed with formalin ${ }^{39,40}$, suggesting that formalin fixation was not the determinant for pathogenic inflammation. Previous studies had shown that FI-RSV induced a predominant $\mathrm{T}_{\mathrm{H}} 2$ cell-like cytokine profile, such as interleukin-5 (IL-5) and IL-13, whereas live RSV, which did not cause ERD, induced a predominant type $1 \mathrm{~T}$ helper cell $\left(\mathrm{T}_{\mathrm{H}} 1\right.$ cell)-like cytokine profile, such as IL-10 (REFS $\left.{ }^{27,41}\right)$. Furthermore, some live attenuated RSV vaccines and some RSV antigens expressed on viral or DNA vectors did not induce, or only slightly induced, ERD in humans ${ }^{42-44}$. One of the reasons why only certain antigens induce ERD may be that the surface glycoprotein of RSV displays diverse structures, thus inducing different immune responses ${ }^{45-47}$. Indeed, several studies showed that exposed antigenic sites differed between prefusion and postfusion surface proteins and that even antibodies targeting a shared site might not bind equally to both conformations $s^{48}$. Notably, another study reported that both postfusion and prefusion $\mathrm{F}$ proteins protected vaccinated cotton rats as long as the antigen concentration was high and the vaccine contained a $\mathrm{T}_{\mathrm{H}} 1$ cell-biasing adjuvant ${ }^{49}$. Aside from the $\mathrm{T}_{\mathrm{H}} 2$ cell-skewed immune response, antibody-mediated effects can also contribute to ERD. The non-NAbs induced by FI-RSV bound antigen, and the antibody-antigen complexes then stimulated the complement pathway, thus further strengthening the inflammatory responses ${ }^{50}$.

In 2019, an RSV vaccine based on an adenovirus vector expressing RSV $\mathrm{F}$ protein stabilized in its prefusion conformation (Ad26.RSV.preF) passed the FDA Breakthrough Therapy Designation programme for the prevention of RSV in older adults. Ad26.RSV.preF induced a high titre of $\mathrm{NAb}$ and long-lasting $\mathrm{T}_{\mathrm{H}} 1$ cell-biased immunity characterized by a high ratio of interferon- $\gamma\left(\mathrm{T}_{\mathrm{H}} 1\right.$-type cytokine) and $\mathrm{T}_{\mathrm{H}}$ 2-type cytokines (IL-4, IL-5 or IL-10) in adult and neonatal mice ${ }^{51}$. However, the clinical trial of Ad26.RSV. preF was done only in adults aged 60 years or older ${ }^{52}$; thus, an RSV vaccine for infants remains elusive. Thus, throughout the 50 -year history of exploring RSV vaccines, we have learnt the absolute necessity of tracking the comprehensive safety of vaccines before large-scale application, no matter the urgency of the moment. From the RSV experience, we still do not know what features of an antigen will create disease exacerbation, although we do know that antigen conformation and prefusion versus fusion states are important. We have also learnt that a $\mathrm{T}_{\mathrm{H}} 2$ cell-biased immune response is harmful. For example, an antigen-induced $\mathrm{T}_{\mathrm{H}} 2$ cell-like cytokine profile, such as IL-5 and IL-13, could activate $\mathrm{CD}^{+} \mathrm{T}$ cells but poorly stimulate natural killer cells and $\mathrm{CD} 8^{+} \mathrm{T}$ cells in an animal model or human. Such a $\mathrm{T}_{\mathrm{H}} 2$ cell-biased immune response might result in VADE under viral challenge. Furthermore, we have learnt that the induction of NAbs over binding antibodies is crucial.

\section{Lessons from dengue vaccines}

Similarly to RSV, the development of dengue vaccines started with an inactivated virus-based vaccine. In the 1920s, Blanc 
and Cminopetros inoculated study participants with a bile-DENV mixture ${ }^{53}$. However, this vaccine failed to protect the participants from subsequent DENV challenge. Afterwards, many studies found that natural DENV infection induced high-titre and sustained NAb responses towards homologous DENV in patients ${ }^{54,55}$. A group of researchers obtained an attenuated DENV strain by serial passage of DENV in mouse brains ${ }^{56}$. One dose of the attenuated vaccine was adequate to induce $\mathrm{NAb}$ in vaccinated volunteers. DENV has four serotypes (DENV1-DENV4), which share a considerable similarity in antigenic epitopes. The induced NAbs not only protected the patient from homologous viral infection but were also cross-reactive with heterologous DENVs. However, the latter protection was short-lived at 3 months to
2 years $^{57}$. Importantly, the cross-specific antibodies, once falling into suboptimal concentrations, caused a higher risk of severe dengue symptoms following natural infection with heterologous DENV than in naive individuals ${ }^{58}$.

This phenomenon was widely investigated. The cross-reactive antibodies bound heterologous DENV, thus facilitating viral entry into target cells with FcRs, such

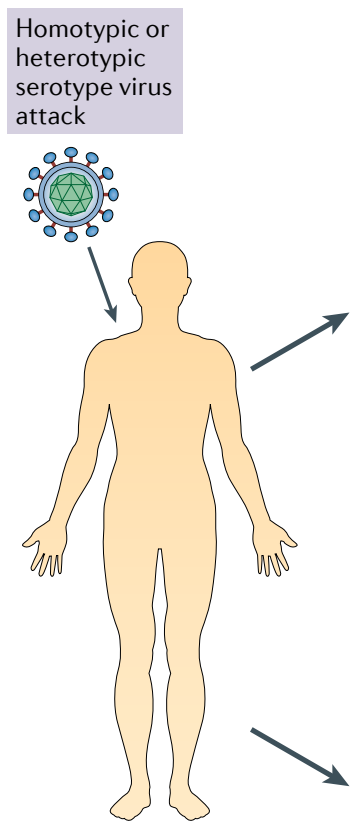

Vaccinated individual

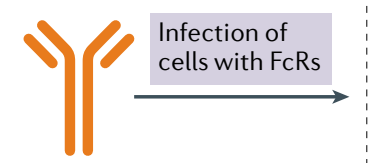

\section{Antibody} (non-neutralizing Abs or neutralizing Abs under suboptimal concentration)

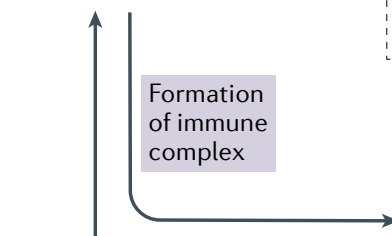

Antibody-dependent enhancement 
as monocytes, macrophages and dendritic cells $^{19,59}$. Meanwhile, epidemiological studies showed that the occurrence of severe dengue was associated with a certain range (DENV antibody titres of 1:21 to 1:80) of cross-reactive antibody titres ${ }^{60,61}$. Aside from entry enhancement, non-NAbs, or NAbs below the optimal concentration, could form complexes with DENV particles, which then induced inflammatory responses through the FcR-mediated immune regulatory pathway $^{62}$, further increasing the risk of severe dengue.

It was clear that reinfection by heterotypic serotype DENV resulted in ADE. Therefore, the next challenge in dengue vaccine development was the induction of NAbs against all four DENV serotypes. It was not until 2006, 77 years after the first inactivated dengue vaccine had been tested in humans, that the first tetravalent dengue vaccine, CYD-TDV, entered clinical trials (NCT00384670). CYD-TDV is a recombinant, live attenuated vaccine with four serotypes of DENV expressed on the yellow fever backbone $^{63}$. In 2018, the FDA approved the CYD-TDV vaccine for preventing dengue caused by all serotypes (DENV1-DENV4). However, administration of this vaccine was not permitted in individuals not previously infected with DENV. This decision was made because clinical analyses revealed an excess risk of severe dengue in seronegative vaccinated individuals compared with seronegative non-vaccinated individuals ${ }^{64}$.

Because DENV can infect FcR-bearing cells whereas SARS-CoV-2 cannot, ADE of viral infection and disease may be more prominent in dengue than in COVID-19, in which it might be milder or even absent. Besides, the pathophysiology of dengue is not comparable to that of COVID-19; thus, the VADE mechanisms of DENV are possibly not related to those in SARS-CoV-2. Still, valuable lessons can be learnt from the long and challenging task of developing a dengue vaccine. First, aside from neutralizing activity, we know that the titre of antibodies induced by any vaccine should be fully evaluated. Low titres of NAbs caused ADE in subsequent infection, instead of providing protection, as observed in both DENV infection ${ }^{58}$ and RSV infection ${ }^{50}$. Second, population genetic analyses of 103 SARS-CoV-2 genomes indicated that SARS-CoV-2 had evolved into two major types (L and S) based on different gene mutations in ORF1ab and ORF8 (REF ${ }^{65}$ ). A further study discovered a 382-nucleotide deletion in ORF8 during the early evolution of SARS-CoV-2 (REF ${ }^{66}$ ). The SARS-CoV-2 variant carrying a D614G alteration in the $S$ protein became the most prevalent in the global pandemic ${ }^{67}$. More than six human coronaviruses are prevalent in human populations, and many more are prevalent in wild animal species. It is unclear so far whether the continuing mutation and recombination of SARS-CoV-2 could create other serotypes of SARS-CoV-2, or even another novel coronavirus. Therefore, vaccine candidates that can provide protection from divergent coronaviruses would be ideal. Third, clinical data from a large cohort revealed that dengue vaccine performance and efficacy could be influenced by the serotype, baseline serostatus and age ${ }^{63,68}$. These results constitute a warning that COVID-19 vaccine candidates should be comprehensively assessed in diverse animal models (that is, young and old animals, and male and female animals) to confirm their safety and efficacy and that human study participants should reflect diverse populations. This is further underscored by the different COVID-19 severity according to age and sex, with older and male individuals at higher risk of severe disease during primary infection ${ }^{69}$.

\section{Lessons from SARS and MERS vaccines}

The genomes of SARS-CoV-2 and SARS-CoV share $79.6 \%$ sequence identity $^{70}$, and they use the same receptor, angiotensin-converting enzyme 2

(ACE2), to enter cells ${ }^{71}$. Therefore, SARS vaccine-induced immune responses, which have already been studied, would be useful in the evaluation of COVID-19 candidate vaccines. In 2003, soon after isolation of SARS-CoV viral particles and release of the viral genome sequence, SARS vaccine design began. Similarly to COVID-19 vaccine developers, researchers first sought SARS vaccines based on inactivated virus, recombinant subunit proteins and recombinant vectors. Also in 2003, an Ad5 vector-based vaccine that expresses the SARS-CoV S1 protein, membrane (M) protein and nucleocapsid $(\mathrm{N})$ protein was tested in rhesus macaques. These vaccines induced SARS-CoV-specific T cell and $\mathrm{NAb}$ responses ${ }^{72}$. Ad5-SARS-CoV-S led to a substantial reduction in viral load and prevented severe pneumonia in ferrets ${ }^{73}$. A recombinant modified vaccinia virus Ankara vector expressing SARS-CoV S protein elicited a rapid and vigorous NAb response in ferrets; however, a strong inflammatory response in the liver of immunized ferrets occurred after challenge with SARS-CoV ${ }^{74,75}$. More studies then demonstrated that SARS vaccines, based on either inactivated virus or a recombinant vector, could induce eosinophils and $\mathrm{T}_{\mathrm{H}} 2$ cell-skewed immune responses on subsequent challenge with SARS-CoV in a mouse model $^{76-78}$, which is reminiscent of RSV vaccine-induced ERD in infants. Similarly, an inactivated SARS-CoV vaccine and a SARS-CoV S protein-derived peptide vaccine both induced severer lung damage in rhesus macaques after SARS-CoV challenge $^{79}$. A DNA vaccine encoding the $S$ protein of SARS-CoV induced $\mathrm{CD}^{+}$and $\mathrm{CD}^{+} \mathrm{T}$ cell and $\mathrm{NAb}$ responses in a mouse model and in a phase I clinical trial ${ }^{80,81}$.

ADE was also observed in SARS vaccines. A SARS vaccine based on recombinant SARS-CoV S protein protected hamsters from SARS-CoV infection; however, the $S$ protein-specific antibodies could mediate FcR-dependent entry into $\mathrm{B}$ cells in vitro ${ }^{82,83}$. Furthermore, diluted SARS-CoV S protein-specific antibodies resulted in increased virus infectivity and cytopathic effect in an HL-CZ human promonocyte cell line ${ }^{84}$. Except for the $\mathrm{ADE}$, antibody-mediated unbalanced macrophage activation has been reported to be associated with obvious lung injury in vivo. Passive transfer of anti-S IgG abrogated wound-healing responses and promoted proinflammatory monocyte and macrophage recruitment and accumulation in the lungs of macaques after viral challenge, indicating that SARS-CoV S protein-specific antibodies could elicit pathogenic immune responses, as well as enhance disease severity after SARS-CoV infection ${ }^{24}$. Notably, the evidence for anti-S IgG-mediated ADE was observed only in vitro. Therefore, ADE seems a less critical issue than other antibody- and $\mathrm{T}_{\mathrm{H}} 2$ cell-mediated immunopathology in vivo.

MERS-CoV belongs to the genus Betacoronavirus, which also includes SARS-CoV and SARS-CoV-2. Since the virus was first identified in Saudi Arabia in 2012, many vaccine techniques, including subunit vaccines, viral vector and DNA-based vaccines, and inactivated and live attenuated vaccines, have been applied to develop MERS vaccines ${ }^{85}$. Many of them could induce adequate immune responses and protect vaccinated animals from subsequent MERS-CoV infection ${ }^{86}$. However, two studies independently reported that mice vaccinated with inactivated MERS-CoV developed $\mathrm{T}_{\mathrm{H}} 2$ cell-biased immune responses and increased eosinophil infiltrates after viral 
challenge ${ }^{87,88}$. Several lines of evidence have demonstrated that MERS S protein-specific antibodies are able to mediate ADE. A monoclonal antibody induced by recombinant MERS-CoV S1 bound to cell surface IgG FcR and mediated viral entry into HEK293T cells exogenously expressing FcRs and macrophages (induced from THP-1 monocytes) endogenously expressing FcRs through canonical viral receptor-dependent pathways ${ }^{89}$. Rabbits infected with MERS-CoV developed MERS-CoV S protein-specific antibodies without neutralizing activity and protection of animals against reinfection, and concerningly, MERS-CoV-reinfected rabbits showed enhanced pulmonary inflammation associated with complement activation ${ }^{90}$. Overall, signs of VADE are less prominent for MERS vaccines than for SARS vaccines. Currently, one DNA MERS vaccine (INO-4700) and two viral-vectored MERS S protein-based vaccines have shown a favourable safety profile and induced humoral and cellular immune responses against MERS-CoV in phase I clinical trials ${ }^{91-93}$. The VADE phenomena in SARS and MERS vaccine development described above further highlight the lessons we have learnt from RSV and DENV. First, the vaccine candidate for SARS-CoV-2 should induce a balanced $\mathrm{T}$ cell response. Particularly, the $\mathrm{T}_{\mathrm{H}} 1$ cell and $\mathrm{T}_{\mathrm{H}} 2$ cell immune response should be evaluated in animals and humans after vaccination. Second, the phenomenon that only diluted SARS-CoV S protein-specific antibodies resulted in increased viral infectivity ${ }^{84}$ indicates that VADE is related to the antibody titre in immunized subjects.

\section{The putative mechanisms of VADE}

Currently, the mechanisms that underlie VADE have not been clearly defined because its emergence is highly virus, host and antigen specific. However, vaccines have several features in common that can induce VADE in vivo. First, vaccines for infection by viruses that target and replicate in cells with FcRs, including DENV and Ebola virus, are likely to induce $\mathrm{VADE}^{94}$, especially ADE. Up to now, only one study has reported that monocytes, as well as B and T lymphocytes, are susceptible to SARS-CoV-2 active infection, and this report has not been peer-reviewed ${ }^{95}$. Therefore, more effort is needed to relieve this concern. Second, vaccines for infection by viruses that will cause inflammatory damage are likely to result in VADE; for example, SARS-CoV and RSV ${ }^{96}$. About $13.9 \%$ of patients with COVID-19 advanced to severe pneumonia ${ }^{97}$, in which inflammatory responses contributed to pathology. A preliminary report showed that the 28-day mortality was lower in the group of patients with COVID-19 receiving dexamethasone, which has anti-inflammatory effects, plus usual care compared with the patients who received usual care alone in a randomized trial $^{98}$. However, pathology seems highly host specific; thus, no confirmed marker has been identified with the ability to predict which patient will progress to acute respiratory distress syndrome. Similarly, it remains hard to predict which antigen will cause VADE. Third, antigens that elicit non-neutralizing antibodies, or insufficient NAbs, are likely to cause VADE. Several lines of evidence have shown that both RBD-specific IgG and NAbs are detectable in patients recovering from COVID-19 (REFS ${ }^{99,100}$ ). However, both the duration of antibody responses and the potential for long-term protection against subsequent natural infection are unknown. There are disparities in the reported kinetics of antibody responses to SARS-CoV-2 infection. For example, one study reported that "severe infections were associated with earlier seroconversion" ${ }^{101}$, whereas another reported that "delayed, but stronger antibody responses were observed in critical patients" ${ }^{102}$. Besides, two recent cases of reinfection with SARS-CoV-2, in the United States and Ecuador, showed severer symptoms in the second round of infection ${ }^{103,104}$, whereas two reinfection cases in Hong Kong and Europe showed milder symptoms in the second round ${ }^{105,106}$. Notably, the first round of infection did not elicit seroconversion in the patient in Hong Kong, which may be the most critical determinant of the second round of infection. In conclusion, we still do not fully understand the antibody dynamics of patients with COVID-19, and that is why we need to carefully assess the immune responses of vaccine candidates in animal models and clinical trials, which is discussed next.

\section{Implications for COVID-19 vaccines Animal models for evaluation of COVID-19 vaccine safety and efficacy. A vaccine should be highly effective in triggering humoral and cellular responses in vivo because low titres of NAbs ${ }^{58}$ and deficient activation of $\mathrm{CD}^{+}$ T cells ${ }^{12}$ are both risk factors for VADE. Meanwhile, we see two major barriers for the evaluation of safety. First, it usually takes a long time to observe VADE because it appears mainly in subsequent challenge or natural infection, by homologous or heterologous viral strains,}

and the occurrence is often related to antibody titres that have decreased to suboptimal levels ${ }^{47}$. Second, it is unclear whether experimental animals accurately represent human responses. From the experience and lessons derived from past development of RSV, dengue, SARS and MERS vaccines, we offer the following recommendations to developers of a safe and effective COVID-19 vaccine.

First, the safety of COVID-19 vaccine candidates should be evaluated in diverse animal models. As no animal model can accurately mimic the human immune response to vaccine candidates, evaluation in several animal models could avoid the risk of missing pathogenic responses. Second, challenge with heterogeneous viral strains should be applied in COVID-19 vaccine evaluations with antibodies cross-reactive to SARS-CoV and SARS-CoV-2 (REF. ${ }^{107}$ ). Third, experiments should be repeated in the same animal model at different ages. Previous studies proved that dengue vaccine performance and efficacy could be influenced by serotype, baseline serostatus and age ${ }^{63,68} \cdot \mathrm{T}_{\mathrm{H}} 2$ cell-biased immunopathology was observed mainly in ageing mice immunized with inactivated SARS-CoV and alum adjuvant ${ }^{76}$. Venezuelan equine encephalitis virus replicon particles expressing SARS-CoV S protein provided complete short-term protection against heterologous SARS-CoV challenge in young mice, whereas only limited protection was seen in vaccinated senescent animals ${ }^{108}$. Given that older individuals are the population most vulnerable to COVID-19, safety and efficacy assessment in ageing animal models and humans is essential. Fourth, animal experiments and clinical trials should also be performed in animal models and humans with co-morbidities, considering that patients with COVID-19 with co-morbidity were shown to have poorer clinical outcomes than those without, and increasing co-morbidity correlated with much poorer clinical outcomes ${ }^{109}$.

\section{Parameters for evaluating COVID-19} vaccine safety and efficacy. Previously, several parameters were proposed as essential in the evaluation of coronavirus vaccine safety and efficacy, including the geometric mean titre of NAbs, the ratio of NAb titre to non-neutralizing antibody titre, antibody affinity, $\mathrm{T}$ cell response profile, virus titres in the upper and lower respiratory tract, and characterization of lung histopathology with immunohistochemistry for viral antigen and immune cell markers ${ }^{110}$. The titre of NAbs 
induced by a vaccine is the most important indicator for efficacy and safety evaluation because NAbs at a suboptimal concentration do not effectively neutralize and may enhance SARS-CoV-2 infection ${ }^{111}$. Moore and Klasse concluded in a review that "it is not known what benchmark serum antibody and NAb titers must be reached for a SARSCoV-2 S-protein vaccine to protect humans. The animal challenge experiments reviewed above suggest that a serum $\mathrm{NAb} \mathrm{ID}_{50}$ titer in the approximate range of 100 to 500 is required for sterilizing immunity" ${ }^{\prime 12}$. We also noticed an absence of detectable SARSCoV RNA in lung tissues of vaccinated mice with serum NAb titres of 1:189 or higher ${ }^{113}$. The FDA recommended that the NAb titres of convalescent plasma for passive therapy be at least 1:160 $\left(\mathrm{REF}^{114}\right)$. Accordingly, we propose that an effective and safe COVID-19 vaccine should be able to induce antiserum in a mouse model with a neutralization titre of at least 1:160 against live SARS-CoV-2 infection. Enhanced eosinophil filtration in the lung is one of the strongest indicators of VADE caused by SARS vaccines ${ }^{76-78}$ or MERS vaccines ${ }^{87,88}$, which should also be monitored when one is evaluating the safety of COVID-19 vaccines after viral challenge or natural viral infection. On the basis of report by Chen et al. ${ }^{115}$, the eosinophil content in the lung of a mouse immunized with a safe SARS vaccine should be less than $5 \%$ of infiltrating cells after viral challenge. Accordingly, we propose that eosinophil infiltrates of $5 \%$ or greater in the lung of a vaccinated mouse after viral challenge should be considered as a putative parameter for VADE. How long the vaccine-induced $\mathrm{NAb}$ response can last is another parameter for evaluation of the safety and efficacy of a vaccine. Seow et al. recently reported that the NAb titre of some recovered patients with a lower peak titre waned to an undetectable level in 2-3 months ${ }^{116}$, indicating that the duration of NAbs may not be long. By contrast, a large-scale study in Iceland demonstrated that antiviral antibodies to SARS-CoV-2 could last for at least 4 months ${ }^{117}$. Another study found that SARS-CoV-2 $S$ protein-specific memory B cells and circulating follicular helper $\mathrm{T}$ cells are positively associated with plasma neutralizing activity ${ }^{118}$. Therefore, these two indicators may be useful for the surveillance of the longevity of immune responses to SARS-CoV-2 after vaccination. Our previous study showed that NAbs in the sera of mice immunized with an RBDbased SARS vaccine can be maintained at a high titre (1:580) for 6 months ${ }^{113}$. Therefore, we propose that $\mathrm{NAb}$ responses elicited by a COVID-19 vaccine should last for at least 6 months in vaccinated mice.

\section{The best antigen for designing a safe and effective COVID-19 vaccine. An} ideal antigen should be selected for the development of a safe and effective COVID-19 vaccine. The $\mathrm{S}$ protein is the major antigen in most COVID-19 vaccine candidates under development as it contains the major neutralizing epitopes and is located on the surface of the viral particle. However, the full-length $S$ protein of SARS-CoV also contains several immunodominant sites that can induce non-neutralizing antibodies, including those associated with ADE, or harmful immune responses ${ }^{78,79,83,84,119,120}$. For example, antibodies targeting the S597-603 epitope, which is located close to the carboxy terminus of the RBD of SARS-CoV $S$ protein, markedly enhanced SARS-CoV infection of Vero E6 cells compared with antibodies from unimmunized macaques ${ }^{79}$. The RBD subunit of SARS-CoV S protein elicited a strong NAb response and protected against SARS-CoV challenge, without obvious VADE, in a mouse model ${ }^{121,122}$. Our previous studies demonstrated that the RBD contains the main neutralizing epitopes in the $S$ protein able to induce higher titres of NAbs, but lower levels of non-neutralizing antibodies, compared with the S1 subunit or full-length S protein ${ }^{123-127}$. SARS-CoV RBD with Alhydrogel (1:25) as an adjuvant induced strong protection without signs of VADE, whereas full-length SARS-CoV S protein induced weak protection and strong VADE in a mouse model ${ }^{115}$. Meanwhile, most NAbs isolated from the serum of coronavirus-infected patients target the RBD ${ }^{128,129}$. Furthermore, the SARSCoV-2 RBD elicited a potent neutralizing response without $\mathrm{ADE}$ in mice ${ }^{130}$. RBDdimer vaccines against COVID-19, SARS or MERS induced NAb responses to the corresponding virus and showed high yields in pilot-scale production ${ }^{131}$. Our recent study demonstrated that a lipid nanoparticleencapsulated RBD-based mRNA COVID-19 vaccine elicited robust $\mathrm{T}$ cell responses and highly potent NAbs against live SARS-CoV-2 infection with an NAb titre of 1:540 at 70 days after boost immunization in mice ${ }^{132}$. These antibodies could also cross-neutralize SARS-CoV pseudoviruses expressing A proteins of human SARS-CoV strains Tor2 and GD03, as well as palm civet strain SZ3, suggesting that this RBD-based mRNA vaccine has potential to be further developed as a safe and effective vaccine to prevent both SARS-CoV-2 and SARS-CoV infection.
Another lipid nanoparticle-encapsulated SARS-CoV-2 RBD-based mRNA vaccine (ARCoV) elicited robust NAbs and $\mathrm{T}_{\mathrm{H}} 1$ cellbiased cellular response in mice and nonhuman primates, while conferring complete protection against mouse-adapted SARS-CoV-2 challenge in the former model ${ }^{133}$.

In addition, several groups have reported the identification of RBD-targeting and cross-reactive antibodies to SARS-CoV and other human coronaviruses, indicating that some conserved epitopes may exist in RBD. A study identified eight RBD-targeted antibodies derived from patients with SERS that neutralized authentic SARS-CoV-2, SARS-CoV and WIV1 coronavirus with half maximal inhibitory concentrations of $0.05-1.4,0.004-0.06$ and $0.076-1.7 \mu \mathrm{g} \mathrm{ml}^{-1}$, respectively ${ }^{134}$. Another study isolated an RBD-specific antibody, S309, from memory $B$ cells of a patient with SARS. It potently neutralized SARS-CoV-2 and SARS-CoV infection $^{135}$. The RBD from a human strain (GD03) and a palm civet strain (SZ16) of SARS-CoV elicited antibodies in rabbits that strongly reacted with and potently neutralized SARS-CoV and SARS-CoV-2, indicating that the RBD can induce cross-neutralizing antibodies to both SARS-CoV and SARS-CoV-2 (REF. ${ }^{136}$ ). These studies further support the development of RBD-based vaccines. Optimization of the RBD by covering the non-neutralizing antibody epitopes with glycosylation ${ }^{137}$ and exposing the NAb epitopes with deglycosylation ${ }^{138}$ is expected to enhance its protective immunity and reduce its potential to induce non-neutralizing antibodies, suggesting that an optimized RBD is an ideal antigen for development of safe and effective COVID-19 vaccines, although other approaches might also turn out to be safe and effective.

\section{Conclusion and prospects}

In May 1796, a little boy was inoculated with the fester from a cowpox-infected patient, thus initiating the history of vaccination. From then on, vaccines have been instrumental in combating many viral diseases, such as smallpox, rabies and polio. The phenomenon of VADE has, however, erected substantial barriers to the development of vaccines for some viruses, including, RSV, DENV, SARS-CoV and MERS-CoV. Currently, the unabated spread of COVID-19 has prompted several countries to rush into local vaccine approval without a comprehensive safety evaluation. Vaccines for viruses with high transmissibility but low case fatality, such as SARS-CoV-2, should usually have a higher 
bar for safety than those for viruses with low transmissibility but high case fatality, such as Ebola virus, because many more healthy individuals will have to use them.

On 15 July 2020, the WHO announced that more than 150 countries are engaged in the COVID-19 Vaccine Global Access (COVAX) initiative, a mechanism designed to guarantee rapid, fair and equitable access to COVID-19 vaccines worldwide ${ }^{139}$. This further raises the safety bar for a COVID-19 vaccine as it should be safe for all people in the world, irrespective of age, gender, race and those with or without co-morbidities. If the adverse reaction rate of a COVID- 19 vaccine is only $1 \%$, about 78 million individuals will be affected if the whole world population is vaccinated. The adverse reaction rate of a COVID-19 vaccine should be kept extremely low if it is distributed globally. The comprehensive safety evaluation in different animal models and clinical trials and rational design of antigens and adjuvants will contribute to lower incidence of VADE.

Shan Su', Lanying Du ${ }^{2}$ and Shibo Jiang (D) $1,2 \otimes$ 'Key Laboratory of Medical Molecular Virology (MOE/MOH/CAM), School of Basic Medical Sciences, Fudan University, Shanghai, China.

${ }^{2}$ Lindsley F. Kimball Research Institute, New York Blood Center, New York, NY, USA.

凶e-mail: shibojiang@fudan.edu.cn https://doi.org/10.1038/s41579-020-00462-y

Published online 16 October 2020

1. Graham, B. S. Rapid COVID-19 vaccine development. Science 368, 945-946 (2020).

2. Jackson, L. A. et al. An mRNA vaccine against SARS-CoV-2 - preliminary report. N. Engl. J. Med. https://doi.org/10.1056/NEJMoa2022483 (2020).

3. Zhu, F. C. et al. Safety, tolerability, and immunogenicity of a recombinant adenovirus type- 5 vectored COVID-19 vaccine: a dose-escalation, open-label, non-randomised first-in-human trial. Lancet 395, 1845-1854 (2020).

4. Thanh, Le,T. et al. The COVID-19 vaccine development landscape. Nat. Rev. Drug. Discov. 19, 305-306 (2020).

5. Vabret, N. et al. Immunology of COVID-19: current state of the science. Immunity 52, 910-941 (2020).

6. Jiang, S. Don't rush to deploy COVID-19 vaccines and drugs without sufficient safety guarantees. Nature 579, 321 (2020)

7. Callaway, E. Coronavirus vaccines: five key questions as trials begin. Nature 579,481 (2020)

8. Corbett, K. S. et al. SARS-CoV-2 mRNA vaccine design enabled by prototype pathogen preparedness. Nature https://doi.org/10.1038/s41586-020-2622-0 (2020).

9. Gao, Q. et al. Rapid development of an inactivated vaccine candidate for SARS-CoV-2. Science https:// doi.org/10.1126/science.abc1932 (2020).

10. Wang, $H$. et al. Development of an inactivated vaccine candidate, BBIBP-CorV, with potent protection against SARS-CoV-2. Cell https://doi.org/10.1016/ j.cell.2020.06.008 (2020)

11. van Doremalen, N. et al. ChAdOx $1 \mathrm{nCoV}-19$ vaccine prevents SARS-CoV-2 pneumonia in rhesus macaques. Nature https://doi.org/10.1038/s41586-020-2608-y (2020).

12. Yu, J. et al. DNA vaccine protection against SARS-CoV-2 in rhesus macaques. Science https:// doi.org/10.1126/science.abc6284 (2020).

13. Folegatti, P. M. et al. Safety and immunogenicity of the ChAdOx $1 \mathrm{nCoV}-19$ vaccine against SARS-CoV-2 a preliminary report of a phase $1 / 2$, single-blind, randomised controlled trial. Lancet https://doi.org/ 10.1016/S0140-6736(20)31604-4 (2020).
14. Mulligan, M. J. et al. Phase $1 / 2$ study of COVID-19 RNA vaccine BNT162b1 in adults Nature https./ doi.org/10.1038/s41586-020-2639-4 (2020).

15. Sahin, U. et al. Concurrent human antibody and TH type T-cell responses elicited by a COVID-19 RNA vaccine. Preprint at medRxiv https://doi.org/10.1101/ 2020.07.17.20140533 (2020).

16. Steffen, T. L. et al. The receptor binding domain of SARS-CoV-2 spike is the key target of neutralizing antibody in human polyclonal sera. Preprint at bioRxiv https://doi.org/10.1101/2020.08.21.261727 (2020).

17. Logunov, D. Y. et al. Safety and immunogenicity of an rAd26 and rAd5 vector-based heterologous prime-boost COVID-19 vaccine in two formulations: two open, non-randomised phase $1 / 2$ studies from Russia. Lancet https://doi.org/10.1016/S01406736(20)31866-3 (2020).

18. Hawkes, R. A. Enhancement of the infectivity of arboviruses by specific antisera produced in domestic fowls. Aust. J. Exp. Biol. Med. Sci. 42, 465-482 (1964).

19. Halstead, S. B. \& O'Rourke, E. J. Dengue viruses and mononuclear phagocytes. I. Infection enhancement by non-neutralizing antibody. J. Exp. Med. 146, 201-217 (1977).

20. Peiris, J. S. \& Porterfield, J. S. Antibody-mediated enhancement of Flavivirus replication in macrophagelike cell lines. Nature 282, 509-511 (1979).

21. Robinson, W. E. Jr., Montefiori, D. C. \& Mitchell, W. M. Antibody-dependent enhancement of human immunodeficiency virus type 1 infection. Lancet $\mathbf{1}$, 790-794 (1988)

22. Ochiai, H. et al. Infection enhancement of influenza A NWS virus in primary murine macrophages by antihemagglutinin monoclonal antibody. J. Med. Virol. 36 217-221 (1992).

23. Lee, W. S., Wheatley, A. K., Kent, S. J. \& DeKosky, B. J. Antibody-dependent enhancement and SARS-CoV-2 vaccines and therapies. Nat. Microbiol. https://doi.org 10.1038/s41564-020-00789-5 (2020)

24. Liu, L. et al. Anti-spike IgG causes severe acute lung injury by skewing macrophage responses during acute SARS-CoV infection. JCI Insight https://doi.org/ 10.1172/jci.insight. 123158 (2019)

25. Vennema, H. et al. Early death after feline infectious peritonitis virus challenge due to recombinant vaccinia virus immunization. J. Virol. 64, 1407-1409 (1990).

26. Weiss, R. C. \& Scott, F. W. Antibody-mediated enhancement of disease in feline infectious peritonitis: comparisons with dengue hemorrhagic fever. Comp. Immunol. Microbiol. Infect. Dis. 4, 175-189 (1981).

27. Waris, M. E., Tsou, C., Erdman, D. D., Zaki, S. R. \& Anderson, L. J. Respiratory synctial virus infection in BALB/c mice previously immunized with formalin inactivated virus induces enhanced pulmonary inflammatory response with a predominant Th2-like cytokine pattern. J. Virol. 70, 2852-2860 (1996).

28. Poland, G. A. Another coronavirus, another epidemic, another warning. Vaccine 38, v-vi (2020)

29. Lane, J. M., Millar, J. D. \& Neff, J. M. Smallpox and smallpox vaccination policy. Annu. Rev. Med. 22, 251-272 (1971)

30. NEJM Group. Effective poliomyelitis vaccines. N. Engl. J. Med. 248, 952-953 (1953)

31. Kim, H. W. et al. Respiratory syncytial virus disease in infants despite prior administration of antigenic inactivated vaccine. Am. J. Epidemiol. 89, 422-434 (1969).

32. Murphy, B. R. et al. Dissociation between serum neutralizing and glycoprotein antibody responses of infants and children who received inactivated respiratory syncytial virus vaccine. J. Clin. Microbiol. 24, 197-202 (1986)

33. Kim, H. W. et al. Cell-mediated immunity to respiratory syncytial virus induced by inactivated vaccine or by infection. Pediatr. Res. 10, 75-78 (1976).

34. Connors, M. et al. Pulmonary histopathology induced by respiratory syncytial virus (RSV) challenge of formalin-inactivated RSV-immunized BALB/c mice is abrogated by depletion of CD4+ T cells. J. Virol. 66 7444-7451 (1992).

35. Connors, M. et al. Enhanced pulmonary histopathology induced by respiratory syncytial virus (RSV) challenge of formalin-inactivated RSV-immunized BALB/c mice is abrogated by depletion of interleukin-4 (IL-4) and IL-10. J. Virol. 68, 5321-5325 (1994).

36. Srikiatkhachorn, A. \& Braciale, T. J. Virus-specific CD8+ T lymphocytes downregulate T helper cell type 2 cytokine secretion and pulmonary eosinophilia during experimental murine respiratory syncytial virus infection. J. Exp. Med. 186, 421-432 (1997).
37. Lee, Y. T. et al. Cellular immune correlates preventing disease against respiratory syncytial virus by vaccination with virus-like nanoparticles carrying fusion proteins. J. Biomed. Nanotechnol. 13, 84-98 (2017).

38. Moghaddam, A. et al. A potential molecular mechanism for hypersensitivity caused by formalin-inactivated vaccines. Nat. Med. 12, 905-907 (2006).

39. Murphy, B. R., Sotnikov, A. V., Lawrence, L. A., Banks, S. M. \& Prince, G. A. Enhanced pulmonary histopathology is observed in cotton rats immunized with formalin-inactivated respiratory syncytial virus (RSV) or purified F glycoprotein and challenged with RSV 3-6 months after immunization. Vaccine 8, 497-502 (1990).

40. Hancock, G. E. et al. Generation of atypical pulmonary inflammatory responses in BALB/c mice after immunization with the native attachment $(G)$ glycoprotein of respiratory syncytial virus. J. Virol. 70, 7783-7791 (1996)

41. Graham, B. S et al Priming immunization determines T helper cytokine mRNA expression patterns in lungs of mice challenged with respiratory syncytial virus. J. Immunol. 151, 2032-2040 (1993).

42. Karron, R. A., Buchholz, U. J. \& Collins, P. L. Live-attenuated respiratory syncytial virus vaccines. Curr. Top. Microbiol. Immunol. 372, 259-284 (2013)

43. Wright, P. F. et al. The absence of enhanced disease with wild type respiratory syncytial virus infection occurring after receipt of live, attenuated, respiratory syncytial virus vaccines. Vaccine $\mathbf{2 5}, \mathbf{7 3 7 2 - 7 3 7 8}$ (2007).

44. Collins, P. L. \& Melero, J. A. Progress in understanding and controlling respiratory syncytial virus: still crazy after all these years. Virus Res. 162, 80-99 (2011)

45. Ngwuta, J. O et al. Prefusion F-specific antibodies determine the magnitude of RSV neutralizing activity in human sera. Sci. Transl Med. 7, 309ra162 (2015).

46. Magro, M. et al. Neutralizing antibodies against the preactive form of respiratory syncytial virus fusion protein offer unique possibilities for clinical intervention. Proc. Natl Acad. Sci. USA 109, 3089-3094 (2012).

47. Fedechkin, S. O. et al. Conformational flexibility in respiratory syncytial virus $\mathrm{G}$ neutralizing epitopes. J. Virol. https://doi.org/10.1128/JVI.01879-19 (2020).

48. Phung, E. et al. Epitope-specific serological assays for RSV: conformation matters. Vaccines https://doi.org/ 10.3390/vaccines 7010023 (2019).

49. Schneider-Ohrum, K. et al. Immunization with low doses of recombinant postfusion or prefusion respiratory syncytial virus $\mathrm{f}$ primes for vaccine-enhanced disease in the cotton rat model independently of the presence of a Th1-biasing (GLA-SE) or Th2-biasing (alum) adjuvant. J. Virol. https://doi.org/10.1128/JVI.02180-16 (2017).

50. Melendi, G. A. et al. C5 modulates airway hyperreactivity and pulmonary eosinophilia during enhanced respiratory syncytial virus disease by decreasing C3a receptor expression. J. Virol. 81, 991-999 (2007)

51. van der Fits, L. et al. Adenovector 26 encoded prefusion conformation stabilized RSV-F protein induces long-lasting Th1-biased immunity in neonatal mice. NPJ Vaccines 5, 49 (2020).

52. Williams, K. et al. Phase 1 safety and immunogenicity study of a respiratory syncytial virus vaccine with an adenovirus 26 vector encoding prefusion $\mathrm{F}$ (Ad26.RSV.preF) in adults aged $>1=60$ years. J. Infect. Dis. 222, 979-988 (2020).

53. Blanc, G. $\&$ Cminopetros, J. Contributions to the study of vaccination against dengue. Bull. Acad. Med. 102 , 40-47 (1929).

54. Soekiman, S. A study on susceptibility of Indonesia colonies of Aedes aegypti and Aedes albopictus mosquitoes to experimental infection with dengue type 3 and chikungunya viruses. Kobe J. Med. Sci. 33, 19-34 (1987)

55. Wahala, W. M. \& Silva, A. M. The human antibody response to dengue virus infection. Viruses 3 , 2374-2395 (2011).

56. Dorrance, W. R. et al. Clinical and serologic response of man to immunization with attenuated dengue and yellow fever viruses. J. Immunol. 77, 352-364 (1956).

57. Snow, G. E., Haaland, B., Ooi, E. E. \& Gubler, D. J. Review article: research on dengue during World War II revisited. Am. J. Trop. Med. Hyg. 91, 1203-1217 (2014).

58. Kliks, S. C., Nimmanitya, S., Nisalak, A \& Burke, D. S. Evidence that maternal dengue antibodies are important in the development of dengue hemorrhagic fever in infants. Am. J. Trop. Med. Hyg. 38, 411-419 (1988). 
59. Halstead, S. B., Nimmannitya, S. \& Cohen, S. N. Observations related to pathogenesis of dengue hemorrhagic fever. IV. Relation of disease severity to antibody response and virus recovered. Yale J. Biol. Med. 42, 311-328 (1970)

60. Katzelnick, L. C. et al. Antibody-dependent enhancement of severe dengue disease in humans. Science 358, 929-932 (2017).

61. Salje, H. et al. Reconstruction of antibody dynamics and infection histories to evaluate dengue risk. Nature 557, 719-723 (2018).

62. Rothman, A. L. Immunity to dengue virus: a tale of original antigenic sin and tropical cytokine storms. Nat. Rev. Immunol. 11, 532-543 (2011).

63. Capeding, M. R. et al. Clinical efficacy and safety of a novel tetravalent dengue vaccine in healthy children in Asia: a phase 3, randomised, observer-masked, placebo-controlled trial. Lancet 384, 1358-1365 (2014)

64. Sridhar, S. et al. Effect of dengue serostatus on dengue vaccine safety and efficacy. N. Engl. J. Med. 379, 327-340 (2018).

65. Tang, X. L. et al. On the origin and continuing evolution of SARS-CoV-2. Natl Sci. Rev. 7, 1012-1023 (2020).

66. Su, Y. C. F. et al. Discovery and genomic characterization of a 382-nucleotide deletion in ORF7b and ORF8 during the early evolution of SARS-CoV-2. mBio 11, e01610-20 (2020).

67. Korber, B. et al. Tracking changes in SARS-CoV-2 spike: evidence that D614G increases infectivity of the COVID-19 virus. Cell https://doi.org/10.1016/ j.cell.2020.06.043 (2020)

68. Villar, L. et al. Efficacy of a tetravalent dengue vaccine in children in Latin America. N. Engl. J. Med. 372 113-123 (2015)

69. Petrilli, C. M. et al. Factors associated with hospital admission and critical illness among 5279 people with coronavirus disease 2019 in New York City: prospective cohort study. BMJ 369, m1966 (2020).

70. Zhou, P. et al. A pneumonia outbreak associated with a new coronavirus of probable bat origin. Nature $\mathbf{5 7 9}$ 270-273 (2020).

71. Walls, A. C. et al. Structure, function, and antigenicity of the SARS-CoV-2 spike glycoprotein. Cell https:// doi.org/10.1016/j.cell.2020.02.058 (2020).

72. Gao, W. et al. Effects of a SARS-associated coronavirus vaccine in monkeys. Lancet 362, 1895-1896 (2003).

73. Kobinger, G. P. et al. Adenovirus-based vaccine prevents pneumonia in ferrets challenged with the SARS coronavirus and stimulates robust immune responses in macaques. Vaccine 25, 5220-5231 (2007).

74. Weingartl, H. et al. Immunization with modified vaccinia virus Ankara-based recombinant vaccine against severe acute respiratory syndrome is associated with enhanced hepatitis in ferrets. J. Virol. 78, 12672-12676 (2004).

75. Czub, M., Weingartl, H., Czub, S., He, R. \& Cao, J. Evaluation of modified vaccinia virus Ankara based recombinant SARS vaccine in ferrets. Vaccine 23 2273-2279 (2005)

76. Bolles, M. et al. A double-inactivated severe acute respiratory syndrome coronavirus vaccine provides incomplete protection in mice and induces increased eosinophilic proinflammatory pulmonary response upon challenge. J. Virol. 85, 12201-12215 (2011)

77. Yasui, F. et al. Prior immunization with severe acute respiratory syndrome (SARS)-associated coronavirus (SARS-CoV) nucleocapsid protein causes severe pneumonia in mice infected with SARS-CoV. J. Immunol. 181, 6337-6348 (2008).

78. Tseng, C. T. et al. Immunization with SARS coronavirus vaccines leads to pulmonary immunopathology on challenge with the SARS virus. PLOS ONE 7, e3542 (2012).

79. Wang, Q. et al. Immunodominant SARS coronavirus epitopes in humans elicited both enhancing and neutralizing effects on infection in non-human primates. ACS Infect. Dis. 2, 361-376 (2016).

80. Yang, Z. Y. et al. A DNA vaccine induces SARS coronavirus neutralization and protective immunity in mice. Nature 428, 561-564 (2004).

81. Martin, J. E. et al. A SARS DNA vaccine induces neutralizing antibody and cellular immune responses in healthy adults in a phase I clinical trial. Vaccine $\mathbf{2 6}$, 6338-6343 (2008)

82. Kam, Y. W. et al. Antibodies against trimeric $S$ glycoprotein protect hamsters against SARS-CoV challenge despite their capacity to mediate FcgammaRII-dependent entry into B cells in vitro. Vaccine 25, 729-740 (2007).
83. Yip, M. S. et al. Antibody-dependent infection of human macrophages by severe acute respiratory syndrome coronavirus. Virol. J. 11, 82 (2014).

84. Wang, S. F. et al. Antibody-dependent SARS coronavirus infection is mediated by antibodies against spike proteins. Biochem. Biophys. Res. Commun. 451, 208-214 (2014).

85. Yong, C. Y., Ong, H. K., Yeap, S. K., Ho, K. L. \& Tan, W. S. Recent advances in the vaccine development against middle east respiratory syndrome-coronavirus. Front. Microbiol. 10, 1781 (2019).

86. Du, L., Tai, W., Zhou, Y. \& Jiang, S. Vaccines for the prevention against the threat of MERS-CoV. Expert. Rev. Vaccines 15, 1123-1134 (2016)

87. Agrawal, A. S. et al. Immunization with inactivated middle east respiratory syndrome coronavirus vaccine leads to lung immunopathology on challenge with live virus. Hum. Vaccin. Immunother. 12, 2351-2356 (2016).

88. Li, K. et al. Single-dose, intranasal immunization with recombinant parainfluenza virus 5 expressing middle east respiratory syndrome coronavirus (MERS-CoV) spike protein protects mice from fatal MERS-CoV infection. mBio https://doi.org/10.1128/mBio.00554-20 (2020).

89. Wan, Y. et al. Molecular mechanism for antibody dependent enhancement of coronavirus entry. J. Virol. https://doi.org/10.1128/JVI.02015-19 (2020).

90. Houser, K. V. et al. Enhanced inflammation in New Zealand white rabbits when MERS-CoV reinfection occurs in the absence of neutralizing antibody. PLOS Pathog. 13, e1006565 (2017).

91. Modjarrad, K. et al. Safety and immunogenicity of an anti-Middle East respiratory syndrome coronavirus DNA vaccine: a phase 1, open-label, single-arm, dose-escalation trial. Lancet Infect. Dis. 19, 1013-1022 (2019).

92. Koch, T. et al. Safety and immunogenicity of a modified vaccinia virus Ankara vector vaccine candidate for Middle East respiratory syndrome: an open-label, phase 1 trial. Lancet Infect. Dis. 20, 827-838 (2020).

93. Folegatti, P. M. et al. Safety and immunogenicity of a candidate Middle East respiratory syndrome coronavirus viral-vectored vaccine: a dose-escalation, open-label, non-randomised, uncontrolled, phase 1 trial. Lancet Infect. Dis. 20, 816-826 (2020).

94. Takada, A., Feldmann, H., Ksiazek, T. G. \& Kawaoka, Y. Antibody-dependent enhancement of Ebola virus infection. J. Virol. 77, 7539-7544 (2003)

95. Pontelli, M. C. et al. Infection of human Iymphomononuclear cells by SARS-CoV-2. Preprint at bioRxiv https://doi.org/10.1101/2020.07.28.225912 (2020).

96. Culley, F. J., Pennycook, A. M., Tregoning, J. S. Hussell, T. \& Openshaw, P. J. Differential chemokine expression following respiratory virus infection reflects Th1 - or Th2-biased immunopathology. J. Virol. 80 , 4521-4527 (2006).

97. Borges do Nascimento, I. J. et al. Novel coronavirus infection (COVID-19) in humans: a scoping review and meta-analysis. J. Clin. Med. https://doi.org/10.3390/ jcm9040941 (2020).

98. DTB Team. Dexamethasone for COVID-19: preliminary findings. Drug Ther. Bull. 58, 133 (2020).

99. To, K. K. et al. Temporal profiles of viral load in posterior oropharyngeal saliva samples and serum antibody responses during infection by SARS-CoV-2: an observational cohort study. Lancet Infect. Dis. 20, 565-574 (2020)

100. Ni, L. et al. Detection of SARS-CoV-2-specific humoral and cellular immunity in COVID-19 convalescent individuals. Immunity https://doi.org/10.1016/ j.immuni.2020.04.023 (2020)

101. Young, B. E. et al. Viral dynamics and immune correlates of COVID-19 disease severity. Clin. Infect. Dis. https://doi.org/10.1093/cid/ciaa1 280 (2020).

102. Qu, J. et al. Profile of IgG and IgM antibodies against severe acute respiratory syndrome coronavirus 2 (SARS-CoV-2). Clin. Infect. Dis. https://doi.org/ 10.1093/cid/ciaa489 (2020)

103. Bowen, T. Nevada State Public Health Lab-led team studying COVID-19 reinfection https://med.unr.edu/ news/archive/2020/covid-19-reinfection?_ga= 2.187802988.514921482.15987184071669011675.1598718407 (2020)

104. Belen, P.-V. et al. COVID-19 Re-infection by a phylogenetically distinct SARS-CoV-2 variant, first confirmed event in South America. Preprint at SSRN https://doi.org/10.2139/ssrn.3686174 (2020).

105. To, K. K. et al. COVID-19 re-infection by a phylogenetically distinct SARS-coronavirus-2 strain confirmed by whole genome sequencing. Clin. Infect. Dis. https://doi.org/10.1093/cid/ciaa1275 (2020).

106. Van Elslande, J. et al. Symptomatic SARS-CoV-2 reinfection by a phylogenetically distinct strain. Clin. Infect. Dis. https://doi.org/10.1093/cid/ciaa1330 (2020).

107. Wu, Y. et al. Identification of human single-domain antibodies against SARS-CoV-2. Cell Host Microbe $\mathbf{2 7}$ 891-898 e895 (2020)

108. Deming, D. et al. Vaccine efficacy in senescent mice challenged with recombinant SARS-CoV bearing epidemic and zoonotic spike variants. PLoS Med. 3 , e525 (2006)

109. Guan, W. J. et al. Comorbidity and its impact on 1590 patients with COVID-19 in China: a nationwide analysis. Eur. Respir. J. https://doi.org/10.1183/ 13993003.00547-2020 (2020).

110. Lambert, P. H. et al. Consensus summary report for CEPI/BC March 12-13, 2020 meeting: assessment of risk of disease enhancement with COVID-19 vaccines. Vaccine 38, 4783-4791 (2020)

111. Cao, X COVID-19: immunopathology and its implications for therapy. Nat. Rev. Immunol. 20, 269-270 (2020)

112. Moore, J. P. \& Klasse, P. J. SARS-CoV-2 vaccines: ‘warp speed' needs mind melds not warped minds. J. Virol. https://doi.org/10.1128/JVI.01083-20 (2020).

113. Du, L. et al. Receptor-binding domain of SARS-CoV spike protein induces long-term protective immunity in an animal model. Vaccine 25, 2832-2838 (2007).

114. FDA. Recommendations for Investigational COVID-19 Convalescent Plasma https://www.fda.gov/vaccinesblood-biologics/investigational-new-drug-ind-ordevice-exemption-ide-process-cber/recommendations investigational-covid-19-convalescent-plasma (2020).

115. Chen, W. H. et al. Yeast-expressed SARS-CoV recombinant receptor-binding domain (RBD219-N1) formulated with alum induces protective immunity and reduces immune enhancement. Preprint at bioRxiv https://doi.org/10.1101/2020.05.15.098079 (2020).

116. Seow, J. et al. Longitudinal evaluation and decline of antibody responses in SARS-CoV-2 infection. Preprint at medRxiv https://doi.org/10.1101/ 2020.07.09.20148429 (2020).

117. Gudbjartsson, D. F. et al. Humoral immune response to SARS-CoV-2 in Iceland. N. Engl. J. Med. https:// doi.org/10.1056/NEJMoa2026116 (2020).

118. Juno, J. A. et al. Humoral and circulating follicular helper T cell responses in recovered patients with COVID-19. Nat. Med. https://doi.org/10.1038/ s41591-020-0995-0 (2020)

119. He $\mathrm{Y}$ et al Identification of immunodominant sites on the spike protein of severe acute respiratory syndrome (SARS) coronavirus: implication for developing SARS diagnostics and vaccines. J. Immunol. 173 4050-4057 (2004)

120. Coughlin, M. M. \& Prabhakar, B. S. Neutralizing human monoclonal antibodies to severe acute respiratory syndrome coronavirus: target, mechanism of action, and therapeutic potential. Rev. Med. Virol. 22, 2-17 (2012)

121. Du, L. et al. Intranasal vaccination of recombinant adeno-associated virus encoding receptor-binding domain of severe acute respiratory syndrome coronavirus (SARS-CoV) spike protein induces strong mucosal immune responses and provides long-term protection against SARS-CoV infection. J. Immunol. 180, 948-956 (2008)

122. Jiang, S., Lu, L., Liu, Q., Xu, W. \& Du, L. Receptor binding domains of spike proteins of emerging or re-emerging viruses as targets for development of antiviral vaccines. Emerg. Microbes Infect. 1, e13 (2012).

123. He, Y. et al. Receptor-binding domain of SARS-CoV spike protein induces highly potent neutralizing antibodies: implication for developing subunit vaccine. Biochem. Biophys. Res. Commun. 324, 773-781 (2004).

124. He, Y., Lu, H., Siddiqui, P., Zhou, Y. \& Jiang, S. Receptor-binding domain of severe acute respiratory syndrome coronavirus spike protein contains multiple conformation-dependent epitopes that induce highly potent neutralizing antibodies. J. Immunol. 174 , 4908-4915 (2005).

125. Du, L. et al. The spike protein of SARS-CoV-a target for vaccine and therapeutic development. Nat. Rev. Microbiol. 7, 226-236 (2009).

126. He, Y. et al. Cross-neutralization of human and palm civet severe acute respiratory syndrome coronaviruses by antibodies targeting the receptor-binding domain of spike protein. J. Immunol. 176, 6085-6092 (2006). 
127. Jaume, M. et al. SARS CoV subunit vaccine: antibodymediated neutralisation and enhancement. Hong Kong Med. J. 18, 31-36 (2012)

128. Ying, T. et al. Exceptionally potent neutralization of Middle East respiratory syndrome coronavirus by human monoclonal antibodies. J. Virol. 88 , 7796-7805 (2014).

129. Berry, J. D. et al. Neutralizing epitopes of the SARS-CoV S-protein cluster independent of repertoire, antigen structure or mAb technology. mAbs 2, 53-66 (2010).

130. Quinlan, B. D. et al. The SARS-CoV-2 receptor-binding domain elicits a potent neutralizing response without antibody-dependent enhancement. Preprint at bioRxiv https://doi.org/10.1101/2020.04.10.036418 (2020).

131. Dai, L. et al. A universal design of betacoronavirus vaccines against COVID-19, MERS, and SARS. Cell https://doi.org/10.1016/j.cell.2020.06.035 (2020).

132. Tai, W. et al. A novel receptor-binding domain (RBD) based mRNA vaccine against SARS-CoV-2. Cell Res. 30, 932-935 (2020).

133. Zhang, N.-N. et al. A thermostable mRNA vaccine against COVID-19. Cell https://doi.org/10.1016/ j.cell.2020.07.024 (2020)
134. Wec, A. Z. et al. Broad neutralization of SARS-related viruses by human monoclonal antibodies. Science https://doi.org/10.1126/science.abc7424 (2020).

135. Pinto, D. et al. Cross-neutralization of SARS-CoV-2 by a human monoclonal SARS-CoV antibody. Nature 583, 290-295 (2020).

136. Zhu, Y. et al. Cross-reactive neutralization of SARS-CoV-2 by serum antibodies from recovered SARS patients and immunized animals. Preprint at bioRxiv https://doi.org/ 10.1101/2020.04.20.052126 (2020)

137. Du, L. et al. Introduction of neutralizing immunogenicity index to the rational design of MERS coronavirus subunit vaccines. Nat. Commun. 7, 13473 (2016).

138. Chen, W. H. et al. Yeast-expressed recombinant protein of the receptor-binding domain in SARS-CoV spike protein with deglycosylated forms as a SARS vaccine candidate. Hum. Vaccin. Immunother. 10 , 648-658 (2014)

139. WHO. More than 150 countries engaged in COVID-19 vaccine global access facility https://www.who.int news-room/detail/15-07-2020-more-than-150countries-engaged-in-covid-19-vaccine-global-accessfacility (2020).

\section{Acknowledgements}

The authors thank L. Lu at the School of Basic Medical Sciences, Fudan University, for providing his valuable comments and suggestions to improve the manuscript.

\section{Author contributions}

S.J. contributed to discussion of the content, S.S. wrote the article and L.D. and S.J. reviewed and edited the manuscript before submission.

\section{Competing interests}

The authors declare no competing interests.

\section{Peer review information}

Nature Reviews Microbiology thanks Akiko Iwasaki, Vincent Munster, and the other, anonymous, reviewer(s) for their contribution to the peer review of this work.

\section{Publisher's note}

Springer Nature remains neutral with regard to jurisdictional claims in published maps and institutional affiliations.

(c) Springer Nature Limited 2020 